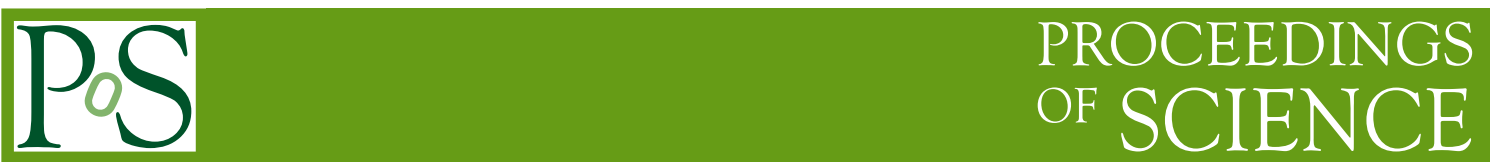

\title{
Maser studies in evolved stars
}

\section{Francisco Colomer*}

Observatorio Astronómico Nacional, Calle Alfonso XII 3, E-28014 Madrid, Spain

E-mail: f.colomerdoan.es

High resolution maps of maser emission provide very detailed information on processes occurring in circumstellar envelopes of late-type stars. A particularly detailed picture of the innermost shells around AGB stars is provided by $\mathrm{SiO}$ masers. Considerable progress is being made to provide astrometrically aligned multi-transition simultaneous observations of these masers, which are needed to better constrain the models. In view of the large amount of high quality data available, models should now be developed to fully explain all maser characteristics together (spatial distribution, variability, etc). New generation instruments (VERA, VSOP-2), new observational techniques (frequency-phase transfer), and new models promise important improvements of our knowledge on this topic.

The 9th European VLBI Network Symposium on The role of VLBI in the Golden Age for Radio Astronomy and EVN Users Meeting

September 23-26, 2008

Bologna, Italy

\footnotetext{
* Speaker.
} 


\section{Introduction}

The evolution of late-type stars is dominated by their mass-loss processes. Asymptotic giant branch stars (AGBs) have developed a large circumstellar envelope (CSE), rich in molecules, which extends to very large distances (up to $1000 \mathrm{AU}$ from the central star, or $10^{18} \mathrm{~cm}$ ). The expansion of this envelope is caused by radiation pressure on dust grains, mainly silicates, which form once the temperature and density have decreased enough (at about $10^{14} \mathrm{~cm}$ ). It is essential to understand what happens in the innermost layers of the circumstellar envelope, before dust is formed, to understand the mechanism of mass loss. Very high resolution mapping of these regions, and of the envelope in general, is possible thanks to radio observations with very long baseline interferometry (VLBI). The best probes to study the physical conditions and dynamics in the different circumstellar shells in O-rich late-type stars are the maser line emissions of the $\mathrm{SiO}, \mathrm{H}_{2} \mathrm{O}$, and $\mathrm{OH}$ molecules, which, mainly in the case of $\mathrm{SiO}$, display very compact structures and high brightness temperatures.

It is well known that AGB stars and envelopes display spherical symmetry, while later evolution into planetary nebulae ( $\mathrm{PNe}$ ) show remarkable bipolar structure. The change from AGB to PNe happens through the proto-planetary nebulae (pPNe) phase, which is very short (lasts about 1000 years only). Studies of objects in this phase should provide the information needed to understand the processes involved in the evolution scenario of these very evolved stars.

STAR molecule formationdust formation photochemical reactions

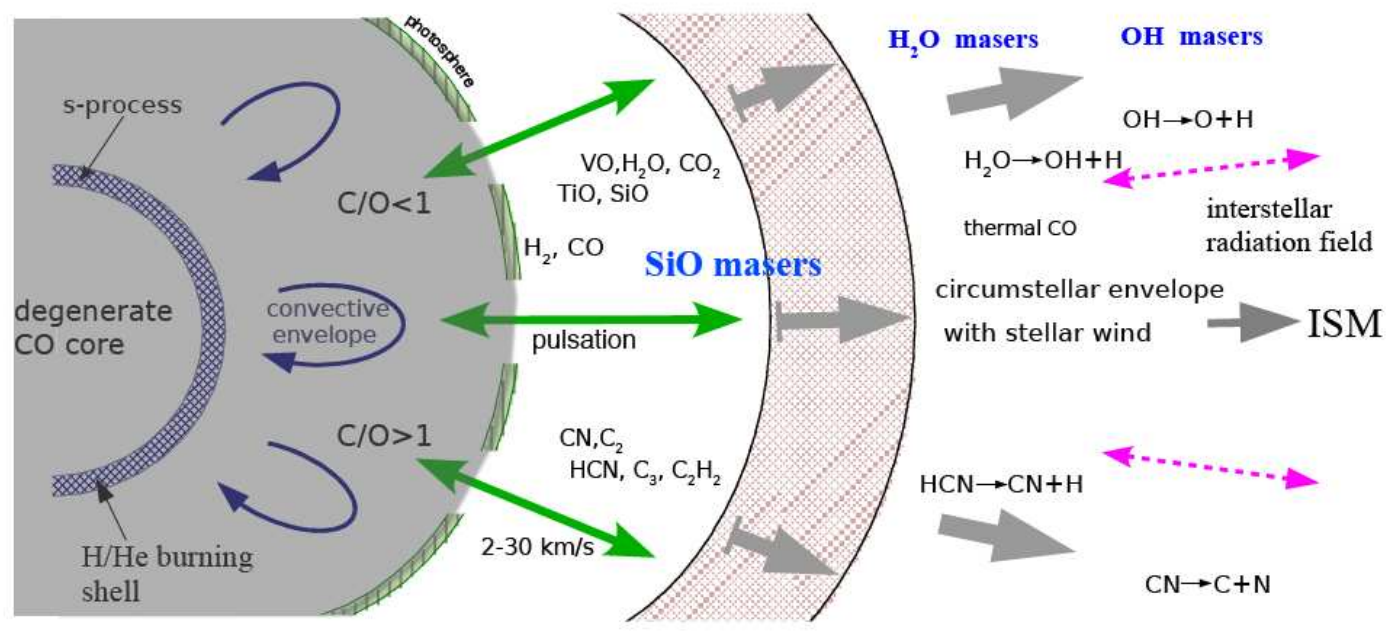

\begin{tabular}{|c|c|c|c|c|}
\hline $10^{8}$ & $10^{13}$ & $10^{14}$ & $r[\mathrm{~cm}]$ & $10^{16}$ \\
\hline $10^{8}$ & 3000 & 1000 & & 100 \\
\hline
\end{tabular}

Figure 1: Structure of a typical AGB circumstellar envelope [1]. 


\section{SiO masers in the inner shells of AGB stars}

$\mathrm{SiO}$ maser emission is a probe of the physical conditions in the inner regions of the star circumstellar envelopes. Because interstellar silicon is incorporated into dust grains (silicates), the dust formation point marks not only the beginning of the expanding wind but also the point where most $\mathrm{SiO}$ disappears from the gas phase, and so happens with its masers.

$\mathrm{SiO}$ masers have been found mostly in oxygen-rich late-type long period variable stars (ratio $[\mathrm{O}] /[\mathrm{C}]>1)$. Positive detections have been reported in the rotational transitions of many excited vibrational states $(0<v \leq 4)$, and on isotopes. Very high spatial resolution observations (using

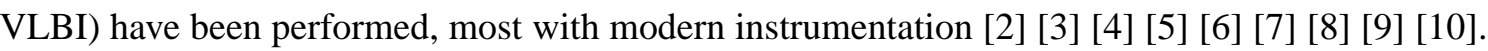
$\mathrm{SiO}$ emission appears as a partial ring structure in most of the objects. The inner envelope kinematics have been revealed by frequent monitoring with the VLBA [11] [12].

The very high quality of the observations mentioned allow to constrain the models of maser emission. The pumping of $\mathrm{SiO}$ masers, for example, has been described to happen by a radiative and/or collisional mechanism; these models predict some features that now can be searched for in the maps. Both expect to find a cascade of masers (i.e. the $J=2 \rightarrow 1$ maser overpopulates the $J=1$ state, therefore favoring the $J=1 \rightarrow 0$ maser). Surprisingly, VLBI maps show that the $v=1$ and $v=2 J=1 \rightarrow 0$ distributions are very similar [ $[$ ] [9], in spite of their very different excitation conditions, while the $v=1 J=2 \rightarrow 1$ shows a significantly different spatial distribution [5] [8] [10]. A difficulty which needs to be overcomed is the alignment of the maps of the different maser lines; strategies have developed from the alignment of the emission centroid [ [ [ to phase tracking between maser spectral lines [7] and, most recently, astrometrical alignment (see e.g. [13] [14]). On the other hand, the very weak $v=2 J=2 \rightarrow 1$ maser emission in O-rich envelopes was the clue to suggest that the frequency overlap of different molecular lines could play an important role [15]. Following this idea, it has been possible to explain both the similar distributions of the $v=1$ and $v=2 J=1 \rightarrow 0$ lines, and the different behaviour of $v=1 J=2 \rightarrow 1$ [5]. Figure 2 is a good summary of these observational properties, including data on the ground state maser of ${ }^{29} \mathrm{SiO} v=0$ $J=1 \rightarrow 0$.

Some results clearly point in favor of a radiative pumping mechanism. The $\mathrm{SiO}$ maser emission in AGB envelopes is well known to be time variable, with a period of several hundred days. It correlates with the IR emission from the central star [16]. A collisional mechanism cannot explain this fact; not even the pulsation of the star travels fast enough to explain this variability [17]. On the other hand, tangential linear polarization is observed in many of these partial rings [6] [9], a fact which is naturally explained by a radiative pumping model [18]. It is worth noting the remarkably sophisticated models developed to integrate collisional pumping mechanisms (see e.g. [19]).

It has been argued that the ring of $\mathrm{SiO}$ emission could be used to estimate the position of the central star, and these being observable at optical wavelenghts, it would be a tool to relate the radio and optical celestial reference frames. The direct detection of continuum radio emission from the stellar photosphere, and the accurate position of the AGB star, was difficult due to its weakness and large (to VLBI standards) size. Recent results are very promissing in this aspect [20], achieving detection of the o Ceti, R Leo, and $\mathrm{W}$ Hya radio photospheres (with respect to the SiO masers at $43 \mathrm{GHz}$ ) with the VLA. 


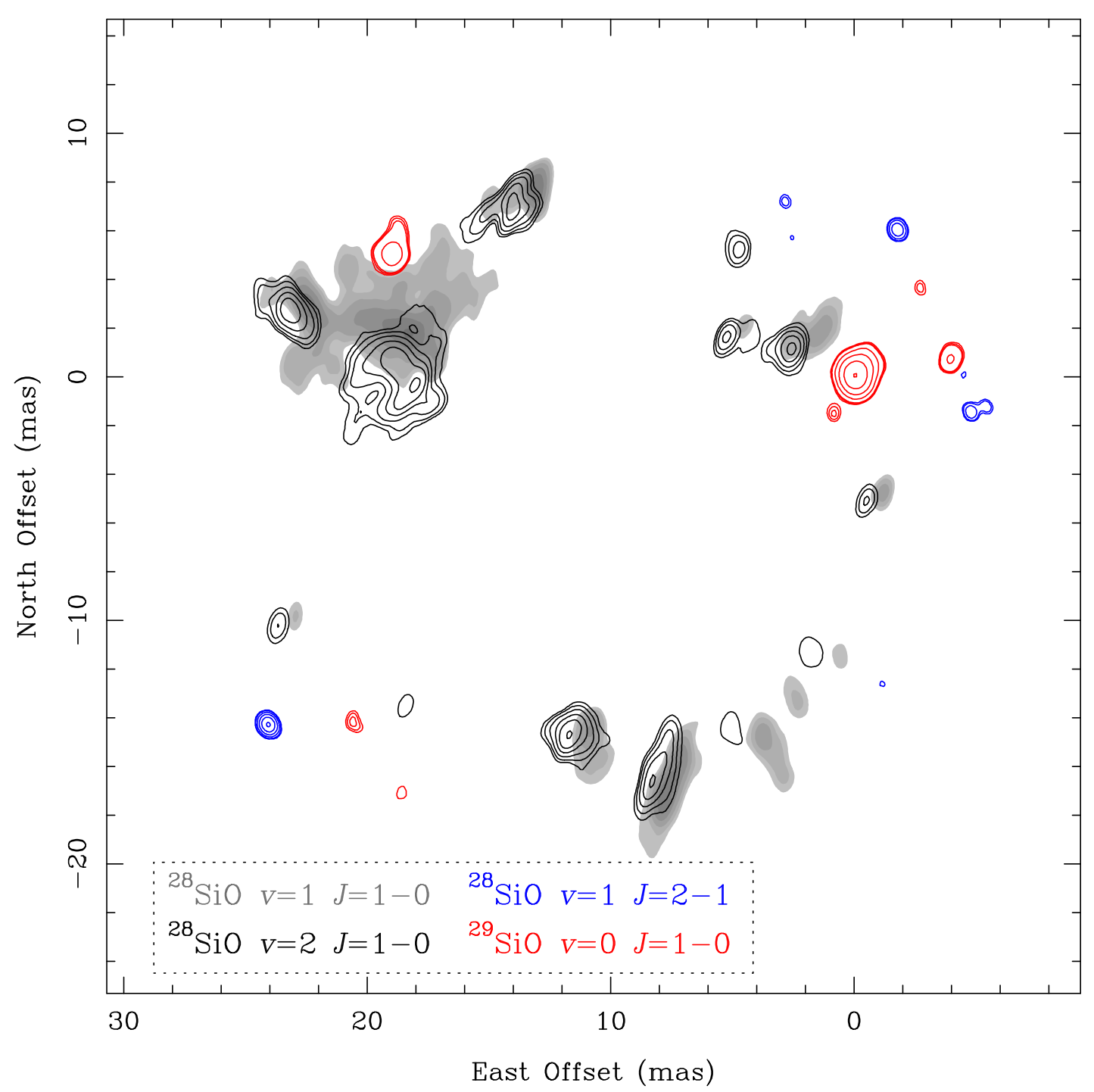

Figure 2: Relative distribution of the $\mathrm{SiO}$ masers around IRC+10011 [1]]

\subsection{Astrometry studies with VERA}

The difficulty in achieving astrometrically aligned $v=1$ and $v=2 \mathrm{SiO}$ maser maps have prevented a unique interpretation of the observations in terms of the physical underlying conditions, which depend on the nature of the $\mathrm{SiO}$ pumping mechanism. The Japanese VLBI Exploration of Radio Astrometry (VERA) project is capable to overcome this limitation by simultaneously observing two sources (reference calibrator and target) separated almost $3^{\circ}$ in the sky. Several AGB stars have been recently investigated in this manner [13] [14] [21] [22]. The newly developed method of frequency-phase transfer calibration has been very useful, in some cases, to relate maser emission of different frequencies.

Thanks to the determination of absolute positions for the SiO maser spots, VERA also provides measurements of the parallax and proper motion [21]. 


\subsection{Results of ultra-high resolution studies}

Ultimate spatial resolution is provided by the global VLBI arrays operating at high frequencies, as well as on space VLBI baselines. To date, the Global Millimeter VLBI Array (GMVA) operating at $86 \mathrm{GHz}$ matches the angular resolution that is expected from the VSOP-2 project at $43 \mathrm{GHz}$, to be launched in 2013. Preliminary detection of ultracompact SiO maser emission with the GMVA [23] indicates that the use of VSOP-2 for these studies is possible. A Key Science Program is being set for the VSOP-2 mission.

\section{The outer shells: $\mathrm{H}_{2} \mathrm{O}$ and $\mathrm{OH}$ masers}

Because $\mathrm{H}_{2} \mathrm{O}$ and $\mathrm{OH}$ masers have a more extended emission and they are very well observed with connected interferometry, studies with VLBI are more scarce.

The VERA instrument is capable to study also the $\mathrm{H}_{2} \mathrm{O}$ masers, and indeed this is its most important design characteristics in order to study our galaxy's structure and rotation by accurate parallax measurements. Mapping of these masers in the envelope of AGB stars have provided some very interesting results [24], for example, a precise determination of the distance to VY CMa [25] [26] which in turn permits to better know its luminosity. The structure and 3-D kinematics of the supergiant star is also revealed, showing a bipolar outflow along the line of sight. Parallax and proper motion for S Crt are also studied in this way [24].

A more complete picture of the AGB envelope is obtained when also the $\mathrm{OH}$ maser emission is included. Combination of MERLIN, EVN/global VLBI data permits to compare the emission of $\mathrm{SiO}, \mathrm{H}_{2} \mathrm{O}$, and $\mathrm{OH}$ masers [27] [28]. The scenario described in Section 1 is challenged, for example in VX Sgr, as mainline $\mathrm{OH}$ masers are found interleaved with $\mathrm{H}_{2} \mathrm{O}$ maser emission. Hints for such overlap are also found in other stars. New studies with coming facilities such as e-MERLIN and ALMA will help imaging the star, trace the dust as it forms, etc.

Observations using absolute phase referencing of the $\mathrm{OH}$ maser peaks, which are expected to coincide with the stellar position, have also been used to measure the absolute coordinates and parallaxes of several AGB stars [29].

\section{Post-AGB objects}

It is well known that, while AGB envelopes are in general quite spherically symmetric, Planetary Nebulae display very different (often beautiful) bipolar structure. The clue for such change must lie in the short-lived protoplanetary nebulae (pPN) phase. Masers are not often observed in pPNe; they have been detected in $\mathrm{OH} 231.8+0.4$ with VLBI ([30]). Absolute positions of $\mathrm{H}_{2} \mathrm{O}$ and $\mathrm{SiO}$ masers have allowed to locate them with great accuracy, also with respect to other components of the nebulae. While the $\mathrm{H}_{2} \mathrm{O}$ emission in this object comes from a region typical of these masers in AGB stars, the bipolar spatial distribution is peculiar, with two spots and $\mathrm{SiO}$ masers in the middle (see Fig. 3). The lack of water in the equatorial regions of the inner circumstellar envelope may be explained by the presence of a companion. MERLIN and VLTI observations of this object complement this scenario [31.

The study of $\mathrm{H}_{2} \mathrm{O}$ and $\mathrm{OH}$ masers in pPNe is very interesing in a particular kind of objects, the "water fountains", sources showing maser spikes at very high velocities. The maser emission in 


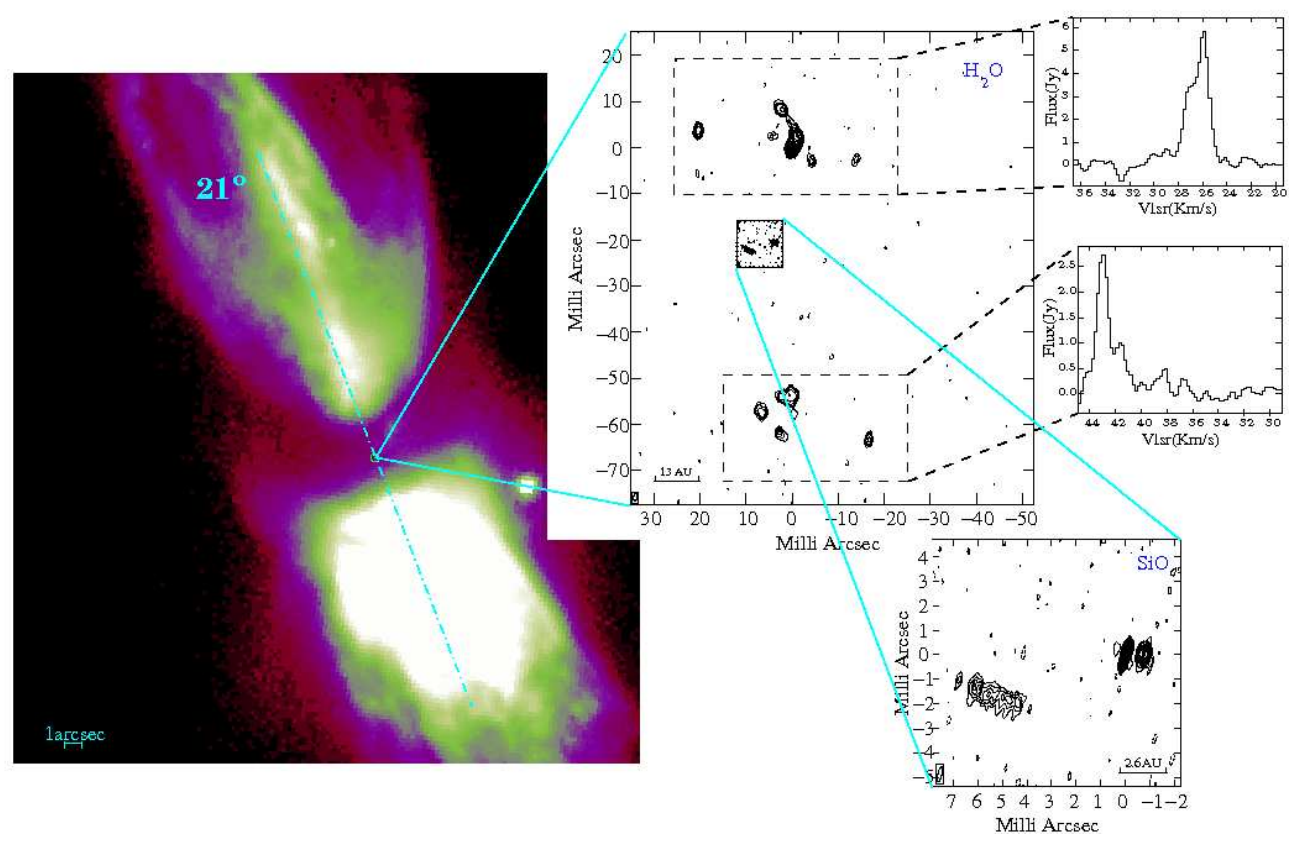

Figure 3: Masers at the heart of pPNe $\mathrm{OH} 231.8+4.2$ [30].

them seems to trace shocks, and proper motions have been measured, see e.g. [32], and references therein.

\section{Summary}

The availability of modern instrumentation has made possible, in the last years, a great advance in the study of maser emission in evolved stars. Nowadays, maps of many $\mathrm{SiO}, \mathrm{H}_{2} \mathrm{O}$, and $\mathrm{OH}$ maser transitions have been produced with very high angular resolution. In many cases, not only the spatial distribution but also the kinematics and polarization of the emission has been revealed.

The position of the emission of the different molecular lines relative to each other is crucial, as it allows to constrain the models in a way that has never happened before. In this respect, new instruments that help to provide absolute astrometry (such as VERA) and new observational techniques (such as frequency-phase transfer) look very promissing to overcome the traditional limitations.

Having so much data available, and with so high quality, it is time to devote special efforts to develop models of maser emission whose predictions fit with the observed maps. Issues such as frequency line overlap should be included in new generation codes. The future looks promissing for the field of maser studies in evolved stars.

\section{Acknowledgements}

I wish to express my gratitude to Valentín Bujarrabal for providing helpful comments to this manuscript. 


\section{References}

[1] R. Soria-Ruiz, 2006, Estudio de Máseres Circunestelares de Monóxido de Silicio con muy alta Resolución Espacial, PhD Thesis, Universidad Autónoma de Madrid, Spain.

[2] P.J. Diamond, A.J. Kemball, W. Junor, A. Zensus, J. Benson, V. Dhawan, Observation of a ring structure in SiO maser emission from late-type stars, 1994, ApJ 430 (61)

[3] L.J. Greenhill, F. Colomer, J.M. Moran, D.C. Backer, W.C. Danchi, W. Bester, 1995, Interferometric Observations of the SiO Masers and Dust Shell of VX Sagittarii, ApJ 440 (619)

[4] J.-F. Desmurs, V. Bujarrabal, F. Colomer, J. Alcolea, 2000, VLBA observations of SiO masers: arguments in favor of radiative pumping mechanisms, A\&A 360 (189)

[5] R. Soria-Ruiz, J. Alcolea, F. Colomer, V. Bujarrabal, J.-F. Desmurs, K. Marvel, P.J. Diamond, 2004, High resolution observations of $\mathrm{SiO}$ masers: Comparing the spatial distribution at 43 and $86 \mathrm{GHz}$, A\&A 426 (131)

[6] W.D. Cotton, B. Mennesson, P.J. Diamond, et al. 2004, VLBA observations of SiO masers towards Mira variable stars, A\&A 414 (275)

[7] J. Yi, R.S. Booth, J.E. Conway, P.J. Diamond, 2005, SiO masers in TX Cam. Simultaneous VLBA observations of two $43 \mathrm{GHz}$ masers at four epochs, A\&A 432 (531)

[8] R. Soria-Ruiz, F. Colomer, J. Alcolea, V. Bujarrabal, J.-F. Desmurs, K.B. Marvel, 2005, First VLBI mapping of circumstellar ${ }^{2} 9 \mathrm{SiO}$ maser emission, A\&A 432 (39)

[9] W.D. Cotton, W. Vlemmings, B. Mennesson, et al. 2006, Further VLBA observations of SiO masers toward Mira variable stars, A\&A 456 (339)

[10] R. Soria-Ruiz, J. Alcolea, F. Colomer, V. Bujarrabal, J.-F. Desmurs, 2007, Mapping the circumstellar $\mathrm{SiO}$ maser emission in R Leonis, A\&A 468 (L1)

[11] I. Gonidakis, P.J. Diamond, A.J. Kemball, 2006, Kinematics of the $v=1 \mathrm{~J}=1 \rightarrow 0 \mathrm{SiO}$ masers at $43 \mathrm{GHz}$ around TX Cam - New 73 epoch movie, in proceedings of Recent Advances in Astronomy and Astorphysics: $7^{\text {th }}$ International Conference of the Hellenic Astronomical Society. AIP Conference Proceedings, Volume 848 (333)

[12] I. Gonidakis, P.J. Diamond, A.J. Kemball, 2009, Kinematics of the $v=1 \mathrm{~J}=1 \rightarrow 0$ SiO masers at $43 \mathrm{GHz}$ around TX Cam - New 73 epoch movie, MNRAS (in press)

[13] M.J. Rioja, R. Dodson, R. Kamohara, F. Colomer, V. Bujarrabal, H. Kobayashi, 2008, Relative Astrometry of the $J=1 \rightarrow 0, v=1$ and $v=2$ SiO Masers towards R Leonis Minoris Using VERA, PASJ 60 (1031) [arXiv: 0811 . 3820]

[14] M.J. Rioja, R. Dodson, F. Colomer, V. Bujarrabal, R. Kamohara, H. Kobayashi, 2009, VERA Astrometric Observations of $43 \mathrm{GHz}$ SiO Masers towards R Leonis Minoris (these proceedings)

[15] H. Olofsson, O.E.H. Rydbeck, A.P. Lane, C.R. Predmore, 1981, Detection of the SiO $v=2$, $J=2 \rightarrow 1$ maser, ApJ 247 (81)

[16] J.R. Pardo, J. Alcolea, V. Bujarrabal, F. Colomer, A. del Romero, P. de Vicente, 2004, ${ }^{28} \mathrm{SiO} v=1$ and $v=2, J=1 \rightarrow 0$ maser variability in evolved stars. Eleven years of short spaced monitoring, A\&A $424(145)$

[17] G.C. McIntosh, 2006, Correlation of Mira's SiO Maser Properties, ApJ 649 (406) 
[18] V. Bujarrabal \& Nguyen-Q-Rieu, 1981, Collisional and radiative excitation of SiO masers, A\&A 102 (65)

[19] E.M.L. Humphreys, M.D. Gray, J.A. Yates, D. Field, G. Bowen, G. P.J. Diamond, 1996, SiO masers in Mira variables at a single stellar phase, MNRAS 282 (1359)

[20] M.J. Reid, K.M. Menten, 2007, Imaging the Radio Photospheres of Mira Variables, ApJ 671 (2068)

[21] R. Kamohara, V. Bujarrabal, T. Bushimata et al. 2008, VERA Observations of SiO Masers in the Symbiotic Star R Aquarii, PASJ 60 (1023)

[22] N. Matsumoto, T. Omodaka, H. Imai et al. 2008, Variable Asymmetry of the Circumstellar Envelope in IK Tauri traced by SiO Maser Emission, PASJ 60 (1039)

[23] F. Colomer, V. Bujarrabal, R. Soria-Ruiz, R. Dodson, J. Alcolea, J.-F. Desmurs, 2009, Detectability of circumstellar $\mathrm{SiO}$ maser emission on VSOP-2 baselines, in proceedings of Approaching Micro-Arcsecond Resolution with VSOP-2: Astrophysics and Technology, Eds. Y. Hagiwara, E. Fomalont, Y. Murata, M. Tsuboi; ASP Conference Series vol 402

[24] A. Nakagawa, M. Tsushima, K. Ando et al. 2008, VLBI Astrometry of AGB Variables with VERA - A Semiregular Variable S Crateris, PASJ 60 (1013)

[25] Y.K. Choi, T. Hirota, M. Honma et al. 2008, Distance to VY Canis Majoris with VERA, PASJ 60 (1007) [arXiv:0808.0641]

[26] Y.K. Choi, T. Hirota, M. Honma, Y. Kobayashi, and VERA members, 2008, Distance to VYCanis Majoris with VERA (these proceedings)

[27] A.M.S. Richards, I. Bains, A. Bartkiewicz, et al. 2007, Turbulent, steamy red supergiant winds, in proceedings of IAU Symposium 242 Astrophysical Masers and their Environments, p. 261

[28] A.M.S. Richards et al. 2009, Maser misto (these proceedings)

[29] W.H.T. Vlemmings, H.J. van Langevelde, 2007, Improved VLBI astrometry of OH maser stars, A\&A 472 (547)

[30] J.-F. Desmurs, J. Alcolea, V. Bujarrabal, C. Sánchez Contreras, F. Colomer, 2007, Water vapor and silicon monoxide maser observations in the protoplanetary nebula OH231.8+4.2, A\&A 468 (189)

[31] S. Etoka, 2009, Structure of the pPN OH231.8+4.2 as revealed by VLTI \& MERLIN interferometric observations, (these proceedings)

[32] M.J. Claussen, R. Sahai, M.R. Morris, 2009, The Motion of Water Masers in the Pre-Planetary Nebula IRAS 16342-3814 2009ApJ...691..219C 